\title{
CORRESPONDENCE
}

\section{Chronic graft-versus-host disease exacerbation after SARS-CoV-2 vaccination}

(c) The Author(s), under exclusive licence to Springer Nature Limited 2022

Bone Marrow Transplantation (2022) 57:502-503; https://doi.org/ 10.1038/s41409-021-01543-z

\section{TO THE EDITOR:}

The gravity of the COVID-19 pandemic compressed the timeline for testing the safety and efficacy of the SARS-CoV-2 vaccines. Therefore, independent evaluation of subgroups of patients that might have different outcomes compared to the unstratified population was not feasible [1]. Consequently, we are learning in real-time how specific patient populations, particularly immunocompromised patients, including those with chronic graft-versushost disease (cGVHD), are affected by the vaccines.

Portuguese et al. reported possible recrudescence of hematologic and autoimmune diseases as a consequence of SARS-CoV-2 vaccination [2]. Thus, it is conceivable that stimulation of the immune system by vaccination or infection could lead to a new presentation or flare of existing CGVHD. A retrospective study involving allogeneic hematopoietic stem cell transplant (allo-HSCT) patients demonstrated worsening of GVHD in 3.5\% of patients, and new GVHD in 9.7\% [3]. In a prospective study, Ram et al. reported worsening of GVHD in $4.5 \%$ of allo-HSCT patients following receipt of the Pfizer SARS-CoV-2 vaccine [4]. Using a similar study design in immunocompromised patients, Bergman et al. observed a worsening of GVHD in $4.4 \%$ of an allo-HSCT patient cohort [5].

Although the immunomodulatory effects of the Pfizer and Moderna products are incompletely understood, vaccine-induced complement activation and stimulation of production of preexisting antibodies have been hypothesized as mechanisms underlying disease exacerbation. The Center for Disease Control and Prevention, the American Society of Hematology, and the American Society of Transplantation and Cellular Therapy recommend the COVID-19 vaccine for immunocompromised patients, including those who have undergone HSCT. Our institution adhered to those recommendations. Herein we report exacerbation of cGVHD in approximately one-fourth of patients who received a SARS-CoV-2 mRNA vaccine.

We performed a retrospective analysis of all patients from our institution who had received allogeneic HSCT between 2013 and 2020. The inclusion criteria for our analysis were patients who had a diagnosis of $C G V H D$ requiring therapy at any time and were alive in December 2020 (when the SARS-CoV-2 vaccine became available). The clinical data was queried from the University of Utah Blood and Marrow Transplant database, and medical records were reviewed for documentation of SARS-CoV-2 mRNA vaccination. Patients meeting the inclusion criteria were screened for new or worsened symptoms of GVHD. The screening process was performed in clinic using standardized system-based questioning for GVHD symptoms, followed by scoring and documentation using an electronic medical record-based flowsheet using National Institutes of Health (NIH) Consensus Criteria for diagnosis and response. We define worsening or flare of disease as an organ increase in severity stage by at least 1 level according to the $\mathrm{NIH}$ Consensus Criteria scale, plus a requirement for systemic and/or topical therapy. All patients were consented under a University of Utah IRB-approved protocol for participation in this study.

Fifty-two patients at our institution met the inclusion criteria. Of these, $34(65.4 \%)$ had two doses of SARS-CoV-2 vaccine and nine of these patients (26.5\%) experienced worsening of cGVHD (Table 1). All nine of these patients had received the Pfizer vaccine. Median time between transplant day 0 and the vaccineassociated flare date was 965 days (95\% Cl, 254-1676). At the time of the flare, $2 / 9$ patients (22.2\%) were on systemic steroids for CGVHD, $7 / 9(77.8 \%)$ had been tapered off of systemic therapy, and three members of this group (33.3\%) were being treated with topical agents (Table 1). Three patients (33.3\%) experienced a cGVHD flare after SARS-CoV-2 vaccine dose \#1. In two of these patients, the flare occurred one day after the initial vaccine treatment (Table 1; Supplementary Fig. 1). The remaining 6 $(66.7 \%)$ experienced a flare after dose \#2, with a median time to flare of 1.5 days after receiving the second injection. No patients experience a flare following both doses \#1 and \#2. Five of the 9 patients $(55.6 \%)$ required systemic steroid therapy, with 2 patients $(22.2 \%)$ requiring $>30$ days of treatment. One patient was enrolled on trial with steroid-sparing systemic therapies. Six patients $(66.7 \%)$ had resolution of flare symptoms. One patient (11.1\%) developed COVID-19 pneumonia and had relapse of AML. The organs most commonly involved were skin $(66.7 \%)$, oropharynx (66.7\%), and eyes (44.4\%). All patients increased organ stage severity by 1 , and in one patient, the score increased by 2 .

Our study suggests that symptom exacerbation following SARSCoV-2 vaccines is relatively common (26.5\%) in allo-HSCT patients who have a history of cGVHD. Though prior reports demonstrated a lower incidence of GVHD flare, those studies analyzed all patients post allo-HSCT, whereas our study focused on cGVHD patients requiring therapy. Our study contributes to the growing body of literature demonstrating that exacerbation, and even new incidence, of CGVHD is a potential adverse effect of COVID-19 vaccination.

The mechanism underlying this process is speculative. Two of 8 patients who were tested were found to have no SARS-CoV-2 IgG spike antibodies, suggesting that post-vaccine flares do not required a brisk immune response to the target antigens of the vaccine (trimerized SARS-CoV-2 receptor binding domain and membraneanchored full-length SARS-CoV-2 spike protein) [6]. On the other hand, the short interval between dose \#2 of the vaccine and onset of symptoms in 5 patients is consistent with other studies in which an immune process (i.e., autoimmunity, molecular mimicry, crossreactivity) appears to drive postvaccination adverse events [7].

While our data suggest that allo-HSCT patients with a history of CGVHD are at risk for disease exacerbation following SARS-CoV-2 
Table 1. Patient characteristics and features of chronic graft-versus-host disease flare.

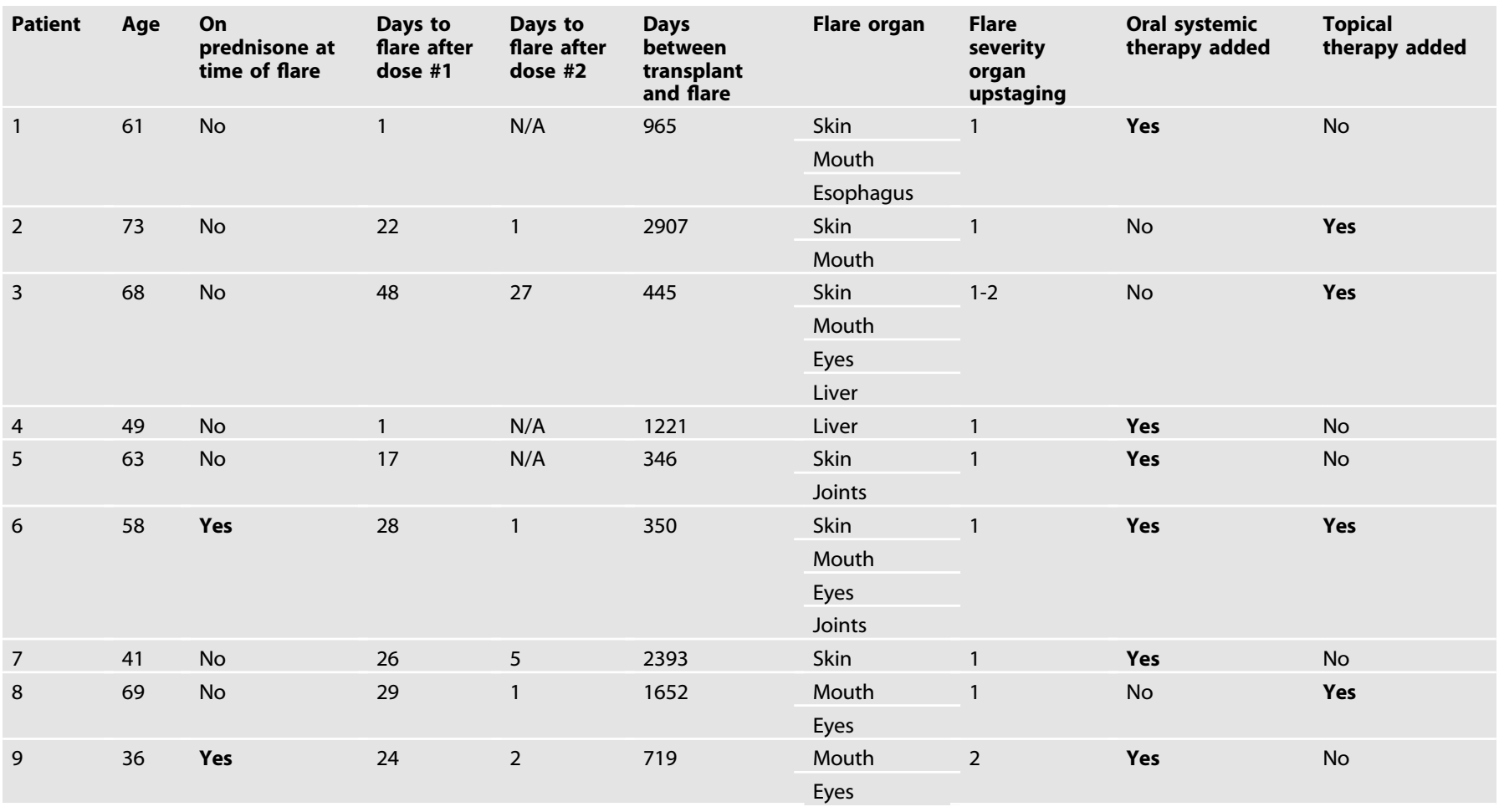

N/A not applicable.

vaccination, we believe that the therapeutic index favors vaccination. Therefore, we continue to recommend that our alloHSCT patients with a history of cGVHD receive the SARS-CoV-2 vaccine; however, we caution them that a CGVHD flare requiring treatment may develop. Following COVID-19 vaccination, careful monitoring of patients with a history of CGVHD is warranted. However, for patients with active GVHD or who experienced an exacerbation of GVHD after the first or second doses of a SARSCoV-2 mRNA vaccine, we recommend deferral of third or booster doses.

\section{Andrew D. Trunk (D) ${ }^{1}$, Samuel K. Shewan (iD) ${ }^{2}$, Catherine J. Lee ${ }^{1}$} Charles J. Parker ${ }^{2}$ and Daniel R. Couriel ${ }^{1 凶}$

${ }^{1}$ The University of Utah Transplant and Cellular Therapy Program, Salt Lake City, UT, USA. ${ }^{2}$ Division of Hematology and Hematologic Maligancies, University of Utah School of Medicine, Salt Lake City, UT, USA. ${ }^{\bowtie}$ email: daniel.couriel@hci.utah.edu

\section{REFERENCES}

1. Sun C, Wiestner A, Pleyer C. COVID-19 vaccines for patients with haematological conditions. Lancet. Haematol. 2021;8:E312-4.

2. Portuguese AJ, Sunga C, Kruse-Jarres R, Gernsheimer T, Abkowitz J. Autoimmuneand complement-mediated hematologic condition recrudescence following SARSCoV-2 vaccination. Blood Adv. 2021;5:2794-8.

3. Ali $H$, Ngo D, Aribi A, Arslan S, Dadwal S, Marcucci G, et al. Safety and tolerability of SARS-CoV-2 Emergency-use authorized vaccines for allogeneic hematopoietic stem cell transplant recipients. 2021. Transplant Cell Ther. 2021; 27:938.e1-938.e6.

4. Ram R, Hagin D, Kikozashvilli N, Freund T, Amit O, Bar-On Y, et al. Safety and immunogenicity of the BNT162b2 mRNA COVID-19 vaccine in patients after allogeneic HCT or CD19-based CART therapy-a single-center prospective cohort study. Transplant Cell Ther. 2021;27:788-94.

5. Bergman P, Blennow O, Hansson L, Mielke S, Nowak P, Chen P, et al. Safety and efficacy of the mRNA BNT162b2 vaccine against SARS-CoV-2 in five groups of immunocompromised patients and healthy controls in a prospective open-label clinical trial. EBioMedicine. 2021;74:103705.
6. Walsh EE, Frenck RW Jr, Falsey AR, Kitchin N, Absalon J, Gurtman A, et al. Safety and immunogenicity of two RNA-based covid-19 vaccine candidates. N Engl J Med. 2020;383:2439-50.

7. Segal Y, Shoenfeld Y. Vaccine-induced autoimmunity: the role of molecular mimicry and immune crossreaction. Cell Mol Immunol. 2018;15:586-94.

\section{ACKNOWLEDGEMENTS}

The authors would like to thank Ed Wicklander and the associated staff involved with curating clinical data in the University of Utah Blood and Marrow transplant database.

\section{AUTHOR CONTRIBUTIONS}

ADT, SKS, CJL, and DRC responsible for project conception, and collection and assembly of data. All authors responsible for data analysis and interpretation, writing and editing of the paper, and approved the final version.

\section{COMPETING INTERESTS}

ADT declares no competing interests. SKS declares no competing interests. CJL has received consulting fees for Jazz and Fresenius Kabi; is on the advisory board and non-promotional Speaker's Bureau for Kite, and advisory board for Kadmon and CareDx; has research funding from Incyte. CJP declares competing interests. DRC has received consulting fees from Incyte and Fresenius Kabi; has been a non-promotional speaker for Mallinckrodt Pharmaceuticals.

\section{ADDITIONAL INFORMATION}

Supplementary information The online version contains supplementary material available at https://doi.org/10.1038/s41409-021-01543-z.

Correspondence and requests for materials should be addressed to Daniel R. Couriel.

Reprints and permission information is available at http://www.nature.com/reprints

Publisher's note Springer Nature remains neutral with regard to jurisdictional claims in published maps and institutional affiliations. 